\title{
Introduction to special section: Best of EuroGP/EvoBio
}

\author{
James A. Foster
}

Published online: 4 July 2013

(c) Springer Science+Business Media New York 2013

This special section comprises two invited articles from a joint session of the 10th European Conference on Evolutionary Computation, Machine Learning, and Data Mining in Computational Biology (EvoBIO 2012) and the 15th European Conference on Genetic Programming (EuroGP 2012). These two conferences met as part of the jointly organized EvoStar (www.evostar.org) event, which was held 11-13 April in Málaga, Spain. This first ever joint session of EuroGP and EvoBIO brought together researchers in genetic programming and researchers using AI inspired algorithms for analyzing biological datasets, to promote mutually beneficial conversations and collaborations. The two papers in this special section are fully revised and significantly extended versions of the research presented at the conference. These papers underwent the same rigorous review and editing process that readers have come to expect from this journal.

The first paper in this special section is "Gene Regulatory Networks Reconstruction from Time Series Datasets using Genetic Programming: a Comparison Between Tree-Based and Graph-Based Approaches," by Leonardo Vanneschi, Matteo Mondini, Martino Bertoni, Alberto Ronchi, and Mattia Stefano. This paper presents two genetic programming approaches to deciphering how genes in biological systems interact to regulate each others' expression, given time series data about which genes are activated in what sequences. Much of the complexity in living organisms derives from these gene regulatory interactions, so it is fundamentally important to form testable hypotheses about expression pathways. Given the size of the relevant datasets, biologists would clearly benefit from an automated approach such as genetic programming. The authors present two GP

James A. Foster-Area Editor for Life Sciences.

\footnotetext{
J. A. Foster ( $\square)$

University of Idaho, Moscow, ID, USA

e-mail: foster@uidaho.edu
} 
systems, GRNGen and GeNet, and show that both are useful, but that only GeNet scales to larger networks.

The second paper is "A New Genetic Programming Framework based on Reaction Systems," by Luca Manzoni, Mauro Castelli, and Leonardo Vanneschi. Rather than using GP to solve an important biological problem, this paper uses biochemistry as a model for a sophisticated new GP methodology. In biological systems, different chemical reagents interact with one another, enhancing or inhibiting a reaction that can only occur when the right ingredients are available in the right quantities. The new approach, called Evolutionary Reaction Systems, models analogous dynamic interactions of computational primitive functions in order to solve a given problem. One potential advantage of this approach is that the GP practitioner need not specify an a priori set of base functions. Rather, the system itself learns the base function set from the dynamics that the programmer describes. The paper presents applications of this idea to several classical test problems as a proof of concept.

These two papers demonstrate the usefulness to both biological and GP research of cross-fertilization between the EvoBIO and EuroGP communities. I predict that there is much more valuable insight to be had from such interactions. In 2014, EvoBIO and EuroGP will again be part of the EvoSTAR conferences, which will meet 23-25 April in Baeza, Jaén, Spain. I invite you to submit your latest work on genetic programming or other approaches to machine learning or data mining for Biological applications to these well-regarded conferences, or just to join us at this important annual cross-disciplinary event. 